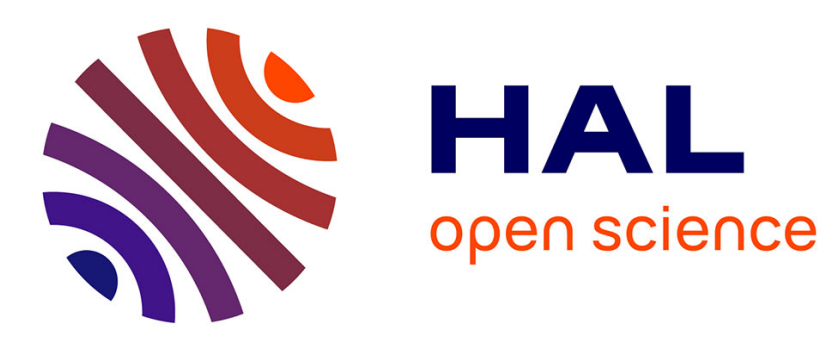

\title{
Adaptive trajectory tracking despite unknown input delay and plant parameters
}

Delphine Bresch-Pietri, Miroslav Krstic

\section{To cite this version:}

Delphine Bresch-Pietri, Miroslav Krstic. Adaptive trajectory tracking despite unknown input delay and plant parameters. Automatica, 2009, 45 (9), pp.2074-2081. hal-00951841

\section{HAL Id: hal-00951841 \\ https://hal.science/hal-00951841}

Submitted on 26 Feb 2014

HAL is a multi-disciplinary open access archive for the deposit and dissemination of scientific research documents, whether they are published or not. The documents may come from teaching and research institutions in France or abroad, or from public or private research centers.
L'archive ouverte pluridisciplinaire $\mathbf{H A L}$, est destinée au dépôt et à la diffusion de documents scientifiques de niveau recherche, publiés ou non, émanant des établissements d'enseignement et de recherche français ou étrangers, des laboratoires publics ou privés. 


\title{
Adaptive Trajectory Tracking Despite Unknown Input Delay and Plant Parameters
}

\author{
Delphine Bresch-Pietri and Miroslav Krstic
}

Department of Mechanical and Aerospace Engineering, University of California, San Diego, La Jolla, CA 92093-0411, USA

\begin{abstract}
In a recent paper we presented the first adaptive control design for an ODE system with a possibly large actuator delay of unknown length. We achieved global stability under full state feedback. In this paper we generalize the design to the situation where, besides the unknown delay value, the ODE also has unknown parameters, and where trajectory tracking (rather than equilibrium regulation) is pursued.
\end{abstract}

\section{Introduction}

Input delay arises in many forms, from an actual physical transport delay in various chemical process systems, hydraulically actuated systems, and combustion systems, to the problems where computational delay manifests itself as being equivalent to input delay. Until recently, the only results on adaptive control of systems with input delays dealt only with uncertain parameters in the ODE part of the system (Evesque et al. 2003, Niculescu and Annaswamy 2003, Ortega and Lozano 1988) but not with uncertainty in the delay value itself. The importance of designing adaptive controllers for unknown delay was recognized in (Kolmanovsky et al. 2001, Krstic and Banaszuk 2006), however only approximation-based ideas for limited classes of plants were dealt with. The robustness of adaptive backstepping control for linear ODEs to dynamic perturbations that include input delay was studied in (Zhou et al. 2008), however the nominal system did not involve delay and the delay was not estimated nor compensated by the controller.

In a recent paper (Bresch-Pietri and Krstic 2009) we presented the first results on delay-adaptive control for a general class of plants, under full state feedback. This result is global-including being global in the initial delay value estimate (one can arbitrarily underestimate or overestimate the delay value initially, and still achieve stabilization adaptively).

However, the result in (Bresch-Pietri and Krstic 2009) contains two limitations, one made for pedagogical reasons and the other which is fundamental. The limitation for which the

Email address: krstic@ucsd.edu (Delphine Bresch-Pietri and Miroslav Krstic). reason is pedagogical is in assuming that there are no unknown parameters in the ODE. This limitation is being removed by this paper. This limitation was imposed in (BreschPietri and Krstic 2009) to prevent the novel ideas on how to develop global adaptivity in the infinite-dimensional (delay) context from being buried under standard but nevertheless complicated details of ODE adaptive control.

The other limitation in (Bresch-Pietri and Krstic 2009), which is fundamental, is in assuming that the full actuator state is measured, though the delay value is completely unknown. The physical meaning of this is that the actuator delay is modeled as a transport process, to which the control designer has physical access for measurement but the speed of propagation of this transport process is completely unknown. As we explain in (Bresch-Pietri and Krstic 2009), the problem where the actuator state is not measurable and the delay value is unknown is not solvable globally, since the problem is not linearly parametrized. We show in (Bresch-Pietri and Krstic 2009) how one can solve it locally, however, this is not a very satisfactory result, since it is local both in the initial state and in the initial parameter error. In other words, the initial delay estimate needs to be sufficiently close to the true delay. (The delay can be long, but it needs to be known quite closely.) Under such an assumption, one might as well use a linear controller and rely on robustness of the feedback law to small errors in the assumed delay value.

So, for this reason, in this paper we continue with a full-state feedback design, specifically assuming the measurement of the actuator state. In (Bresch-Pietri and Krstic 2009) we discussed all the possible problems that one can consider with respect to the availability or unavailability of measurement of the ODE state, the actuator state, the knowledge (or lack of knowledge) of the delay value, and the knowledge (or 
lack thereof) of the ODE parameters. There is a total number of 14 distinct problem combinations. Here we focus on the most interesting one of them, with both the ODE parameters and the delay value unknown, but with full state measurement. An extension to the case where only an output (and not the complete state) of the ODE is available for measurement is easy (with the method of backstepping and Kreisselmeier observers). We don't pursue it here for consistency of concepts - since we must measure the actuator state, we might as well present a result with full measurement of the ODE state.

As in most of the research on control of unstable plants with a long actuator delay (Artstein 1982, Evesque et al. 2003, Fiagbedzi and Pearson 1986, Gu and Niculescu 2003, Jankovic 2006a, Jankovic 2006b, Jankovic 2008, Klamka 1982, Krstic 2008a, Krstic 2008b, Krstic and Smyshlyaev 2008b, Kwon and Pearson 1980, Manitius and Olbrot 1979, Michiels and Niculescu 2003, Michiels and Niculescu 2007, Mirkin 2004, Mondie and Michiels 2003, Niculescu and Annaswamy 2003, Olbrot 1978, Richard 2003, Tadmor 1996, Watanabe and Ito 1996, Watanabe and Ito 1981, Zaccarian and Nesic 2006, Zhong 2006a, Zhong 2006b, Zhong and Mirkin 2002), the essence of our approach is "predictor feedback," which we recently showed in (Krstic 2008a, Krstic and Smyshlyaev 2008b) to be a form of backstepping boundary control for PDEs (Krstic and Smyshlyaev 2008c) and extended to nonlinear plants (Krstic 2008b).

In this paper we generalize the design from (Bresch-Pietri and Krstic 2009) in two major ways: we extend it to ODEs with unknown parameters and extend it from equilibrium regulation to trajectory tracking. A significant number of new technical issues arise in this problem. The estimation error of the ODE parameters appears in the error models of both the ODE and of the infinite-dimensional (delay) subsystem, which is reflected also in the update law. The update law has to also deal appropriately with ensuring stabilizability with the parameter estimates, for which projection is employed. Finally, our approach for dealing with delay adaptation involves normalized Lyapunov-based tuning, a rather non-standard approach as compared to finite-dimensional adaptive control. In this framework, we need to bound numerous terms involving parameter adaptation rates (both for the delay and for the ODE parameters) in the Lyapunov analysis. Some of these terms are vanishing (when the tracking error is zero), while the others (which are due to the reference trajectory) are non-vanishing. These terms receive different treatment though both are bounded by normalization and their size is controlled with the adaptation gain.

We begin in Section 2 by defining the problem and present the adaptive control design and the main stability theorem in Section 3. Simulations results are show in Section 4, which is then followed by the stability proof in Section 5 .

\section{Problem Formulation}

We consider the following system

$\dot{X}(t)=A(\theta) X(t)+B(\theta) U(t-D)$

$Y(t)=C X(t)$

where $X \in \mathbb{R}^{n}$ is the ODE state, $U$ is the scalar input to the entire system, $D>0$ is an unknown constant delay, the system matrix $A(\theta)$ and the input vector $B(\theta)$ are linearly parametrized, i.e.,

$$
\begin{aligned}
& A(\theta)=A_{0}+\sum_{i=1}^{p} \theta_{i} A_{i} \\
& B(\theta)=B_{0}+\sum_{i=1}^{p} \theta_{i} B_{i},
\end{aligned}
$$

and $\theta$ is an unknown but constant parameter vector that belongs to the convex set

$$
\Pi=\left\{\theta \in \mathbb{R}^{p} \mid \mathscr{P}(\theta) \leq 0\right\}
$$

where, by assuming that the convex function $\mathscr{P}: \mathbb{R}^{p} \rightarrow \mathbb{R}$ is smooth, we assure that the boundary $\partial \Pi$ of $\Pi$ is smooth.

Assumption 1 The set $\Pi$ is bounded and known. A constant $\bar{D}$ is known such that $D \in] 0 ; \bar{D}]$.

To make stabilization and tracking possible in the presence of unknown parameters, we make some assumption and illustrate, with the help of an example introduced below, that these assumptions are reasonable. In the sequel, $C^{0}(\Pi)$ and $C^{1}(\Pi)$ is the usual notation for continuous and continuously differentiable functions (on the set $\Pi$ ), respectively.

Assumption 2 We assume that the pair $(A(\theta), B(\theta))$ is completely controllable for each $\theta$. Furthermore, we assume that there exists a triple of vector/matrix-valued functions $(K(\theta), P(\theta), Q(\theta))$ such that $K \in C^{1}(\Pi), P \in C^{1}(\Pi)$, $Q \in C^{0}(\Pi)$, the matrices $P(\theta)$ and $Q(\theta)$ are positive definite and symmetric, and the following Lyapunov equation is satisfied for all $\theta \in \Pi$ :

$$
P(\theta)(A+B K)(\theta)+(A+B K)(\theta)^{T} P(\theta)=-Q(\theta) .
$$

Example 1 Consider the example of a potentially unstable plant

$$
\begin{aligned}
\dot{X}_{1}(t) & =\theta X_{1}(t)+X_{2}(t) \\
\dot{X}_{2}(t) & =U(t-D) \\
Y(t) & =X_{1}(t),
\end{aligned}
$$


where we assume $\Pi=[-\underline{\theta} ; \bar{\theta}]$ and define

$$
\begin{gathered}
A(\theta)=A_{0}+\theta A_{1}=\left(\begin{array}{ll}
0 & 1 \\
0 & 0
\end{array}\right)+\theta\left(\begin{array}{ll}
1 & 0 \\
0 & 0
\end{array}\right) \\
B=B_{0}=\left(\begin{array}{l}
0 \\
1
\end{array}\right) .
\end{gathered}
$$

Using the backstepping method we construct the triple $(K, P, Q)$ as

$K(\theta)=-\left(1+(\theta+1)^{2} \theta+2\right)$

$P(\theta)=\frac{1}{2} Q(\theta)=\left(\begin{array}{cc}1+(1+\theta)^{2} & 1+\theta \\ 1+\theta & 1\end{array}\right)$,

which satisfies the Lyapunov equation (6).

\section{Assumption 3 The quantities}

$$
\begin{aligned}
& \underline{\lambda}=\inf _{\theta \in \Pi} \min \left\{\lambda_{\min }(P(\theta)), \lambda_{\min }(Q(\theta))\right\} \\
& \bar{\lambda}=\inf _{\theta \in \Pi} \lambda_{\max }(P(\theta)) .
\end{aligned}
$$

exist and are known.

Example 2 (Example 1 continued) One can show that the eigenvalues of $P(\theta)$ are

$$
\begin{aligned}
& \lambda_{\max }(P(\theta))=\frac{2+(\theta+1)^{2}+|\theta+1| \sqrt{(\theta+1)^{2}+1}}{2} \\
& \lambda_{\min }(P(\theta))=\frac{1}{\lambda_{\max } P(\theta)},
\end{aligned}
$$

from which $\underline{\lambda}$ and $\bar{\lambda}$ area readily obtained over the set $\Pi=$ $[-\underline{\theta} ; \bar{\theta}]$.

Assumption 4 For a given smooth function $Y^{r}(t)$, there exist known functions $X^{r}(t, \theta)$ and $U^{r}(t, \theta)$, which are bounded in $t$ and continuously differentiable in the unknown argument $\theta$ on $\Pi$, and which satisfy

$$
\begin{aligned}
\dot{X}^{r}(t, \theta) & =A(\theta) X^{r}(t, \theta)+B(\theta) U^{r}(t, \theta) \\
Y^{r}(t) & =C X^{r}(t, \theta) .
\end{aligned}
$$

Example 3 (Example 2 continued) Take $Y^{r}(t)=\sin (t)$. Then, the reference trajectory pair for the state and input is

$$
\begin{aligned}
X^{r}(t, \theta) & =\left(\begin{array}{c}
\sin (t) \\
\cos (t)-\theta \sin (t)
\end{array}\right) \\
U^{r}(t, \theta, D) & =-\sin (t+D)-\theta \cos (t+D) .
\end{aligned}
$$

Both functions are bounded in $t$ and continuously differentiable in $\theta$.

\section{Control Design}

To prepare for our adaptive control design, we represent the plant as

$$
\begin{aligned}
\dot{X}(t) & =A(\theta) X(t)+B(\theta) u(0, t) \\
Y(t) & =C X(t) \\
D u_{t}(x, t) & =u_{x}(x, t), \quad x \in[0,1) \\
u(1, t) & =U(t),
\end{aligned}
$$

where

$$
u(x, t)=U(t+D(x-1)) .
$$

The representation of the pure delay as a transport PDE allows a linear parameterization in the unknown delay $D$.

We consider reference trajectories $X^{r}(t)$ and $U^{r}(t)$, such as described in Assumption 4. Let us introduce the following error variables

$$
\begin{aligned}
\tilde{X}(t) & =X(t)-X^{r}(t, \hat{\theta}) \\
\tilde{U}(t) & =U(t)-U^{r}(t, \hat{\theta}, \hat{D}) \\
e(x, t) & =u(x, t)-u^{r}(x, t, \hat{\theta}),
\end{aligned}
$$

with an estimate $\hat{\theta}$ of the unknown $\theta$. When $D$ and $\theta$ are known, one can show that the control law

$$
\begin{aligned}
U(t)= & U^{r}(t)-K X^{r}(t+D) \\
& +K\left[\mathrm{e}^{A D} X(t)+D \int_{0}^{1} \mathrm{e}^{A D(1-y)} B u(y, t) d y\right]
\end{aligned}
$$

achieves exponential stability of the equilibrium $(\tilde{X}, e)=0$, compensating the effects of the delay $D$.

When $D$ and $\theta$ are unknown, we employ the control law

$$
\begin{aligned}
U(t)= & U^{r}(t, \hat{\theta}, \hat{D})-K(\hat{\theta}) X^{r}(t+\hat{D}, \hat{\theta}) \\
& +K(\hat{\theta})\left[\mathrm{e}^{A(\hat{\theta}) \hat{D}(t)} X(t)\right. \\
& \left.+\hat{D}(t) \int_{0}^{1} \mathrm{e}^{A(\hat{\theta}) \hat{D}(t)(1-y)} B(\hat{\theta}) u(y, t) d y\right],
\end{aligned}
$$

based on the certainty equivalence principle. The update laws for the estimates $\hat{D}$ and $\hat{\theta}$ are chosen based on the Lyapunov analysis (presented in Section 5) as

$$
\begin{aligned}
\dot{\hat{D}}(t) & =\gamma_{1} \operatorname{Proj}_{[0, \bar{D}]}\left\{\tau_{D}(t)\right\} \\
\dot{\hat{\theta}}(t) & =\gamma_{2} \operatorname{Proj}_{\Pi}\left\{\tau_{\theta}(t)\right\},
\end{aligned}
$$

with adaptation gains $\gamma_{1}$ and $\gamma_{2}$ chosen as positive and "small 
enough" and with

$$
\begin{aligned}
\tau_{D}(t)= & -\frac{\int_{0}^{1}(1+x) w(x, t) K(\hat{\theta}) \mathrm{e}^{A(\hat{\theta}) \hat{D}(t) x} d x}{1+\tilde{X}(t)^{T} P(\hat{\theta}) \tilde{X}(t)+b \int_{0}^{1}(1+x) w(x, t)^{2} d x} \\
& \times((A+B K)(\hat{\theta}) \tilde{X}(t)+B(\hat{\theta}) w(0, t)) \\
\tau_{\theta}(t)= & \frac{2 \tilde{X}(t)^{T} P(\hat{\theta}) / b-\int_{0}^{1}(1+x) w(x, t) K(\hat{\theta}) \mathrm{e}^{A(\hat{\theta}) \hat{D}(t) x} d x}{1+\tilde{X}(t)^{T} P(\hat{\theta}) \tilde{X}(t)+b \int_{0}^{1}(1+x) w(x, t)^{2} d x} \\
& \times\left(A_{i} X(t)+B_{i} u(0, t)\right)_{1 \leq i \leq p} .
\end{aligned}
$$

The matrix $\mathrm{P}$ is defined in Assumption 2, the standard projector operators are given by

$$
\begin{gathered}
\operatorname{Proj}_{[0, \bar{D}]}\left\{\tau_{D}\right\}=\tau_{D} \begin{cases}0, & \hat{D}=0 \text { and } \tau_{D}<0 \\
0, & \hat{D}=\bar{D} \text { and } \tau_{D}>0 \\
1, & \text { else }\end{cases} \\
\operatorname{Proj}_{\Pi}\left\{\tau_{\theta}\right\}=\tau_{\theta} \begin{cases}1, & \hat{\theta} \in \Pi^{\circ} \\
I-\frac{\nabla_{\hat{\theta}} \mathscr{P}_{\theta} \nabla_{\theta} T}{\nabla_{\hat{\theta}} \mathscr{P}^{T} \nabla_{\theta} \mathscr{P}}, & \hat{\theta} \in \partial \Pi\end{cases} \\
\text { or } \nabla_{\hat{\theta}} \mathscr{P}^{T} \tau \leq 0
\end{gathered}
$$

The transformed state of the actuator is needed in the update law and is defined as

$$
\begin{aligned}
w(x, t)= & e(x, t)-\hat{D}(t) \int_{0}^{x} K(\hat{\theta}) \mathrm{e}^{A(\hat{\theta}) \hat{D}(t)(x-y)} B(\hat{\theta}) e(y, t) d y \\
& -K(\hat{\theta}) \mathrm{e}^{A(\hat{\theta}) \hat{D}(t) x} \tilde{X}(t)
\end{aligned}
$$

and the constant $\mathrm{b}$ is chosen such as

$$
b \geq 4 \sup _{\hat{\theta} \in \Pi}|P B|^{2}(\hat{\theta}) \frac{\bar{D}}{\underline{\lambda}}
$$

Theorem 1 Let Assumptions 1-4 hold and consider the closed-loop system consisting of (22)-(25), the control law (31) and the update laws defined by (32)-(39). There exists $\gamma^{*}>0$ such that for any $\gamma \in\left[0, \gamma^{*}[\right.$, there exist positive constants $R$ and $\rho$ (independent of the initial conditions) such that, for all initial conditions satisfying $\left(X^{0}, u^{0}, \hat{D}^{0}, \theta^{0}\right) \in$ $\left.\left.\mathbb{R}^{n} \times L_{2}(0,1) \times\right] 0, \bar{D}\right] \times \Pi$, the following holds:

$$
\Upsilon(t) \leq R\left(\mathrm{e}^{\rho \Upsilon(0)}-1\right), \quad \forall t \geq 0
$$

where

$$
\Upsilon(t)=|\tilde{X}(t)|^{2}+\int_{0}^{1} e(x, t)^{2} d x+\tilde{D}(t)^{2}+\tilde{\theta}(t)^{T} \tilde{\theta}(t)
$$
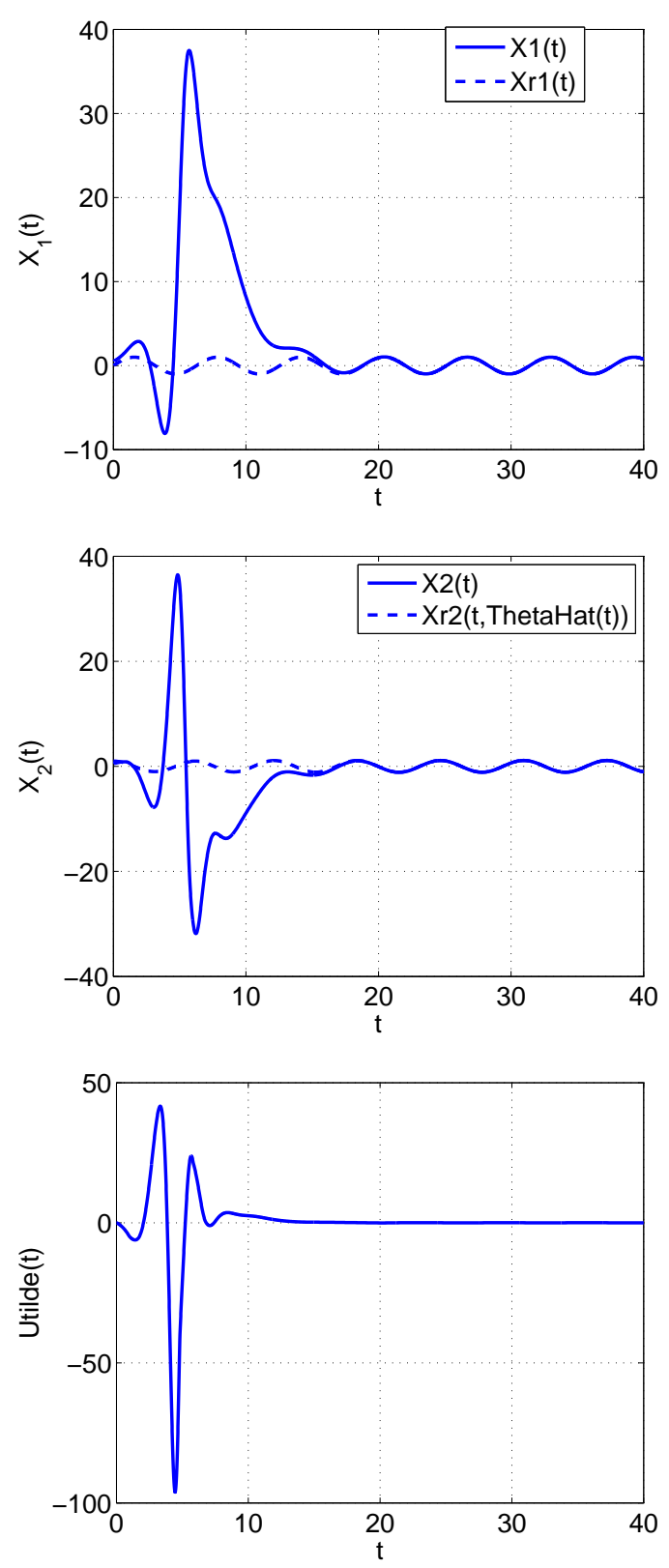

Fig. 1. The system respons of the system (1)-(2) with the reference trajectory (20)-(21) for $D=1, \theta=0.5, \hat{\theta}(0)=0$ and $\hat{D}(0)=0$.

Furthermore, asymptotic tracking is achieve, i.e.,

$$
\lim _{t \rightarrow \infty} \tilde{X}(t)=0, \quad \lim _{t \rightarrow \infty} \tilde{U}(t)=0 .
$$

\section{Simulations}

We return to the system from Examples 1-3 and present simulation results for the closed-loop system consisting of the plant (1)-(2), the control law (31) and the update laws defined through (32)-(39). We focus on the issues arising 

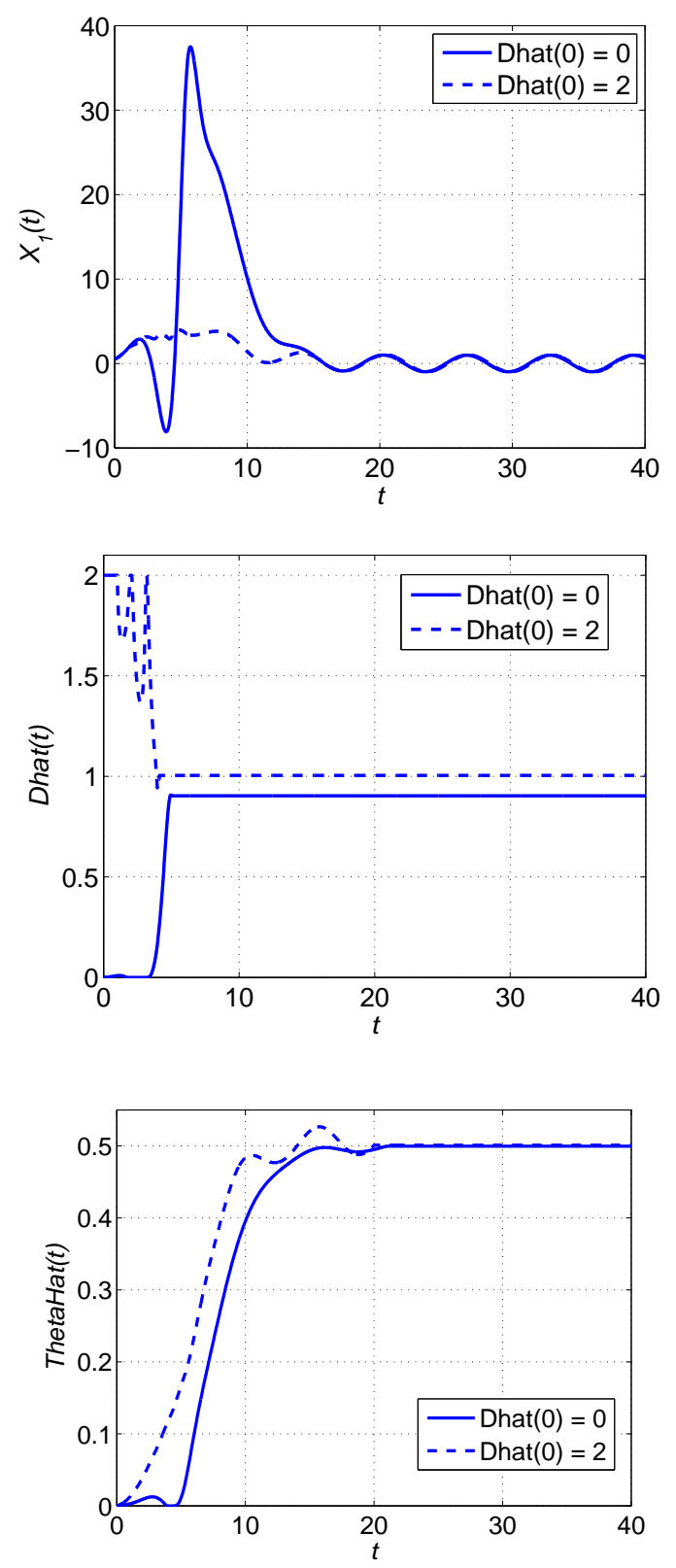

Fig. 2. The system response of the system (1)-(2) with the reference trajectory (20)-(21) for $D=1, \theta=0.5, \hat{\theta}(0)=0$ and two dramatically different initial conditions for $\hat{D}: \hat{D}(0)=0$ and $\hat{D}(0)=\bar{D}=2 D=2$. Note that the solid plots in this figure correspond to the same simulation as in Figure 1.

from the large uncertainties in $D$ and $\theta$ and from the tracking problem with the reference trajectory (20)-(21). We take $D=1, \theta=0.5, \bar{D}=2 D=2, \underline{\theta}=0, \bar{\theta}=2 \theta=1$. We pick the adaptations gains as $\gamma_{1}=10, \gamma_{2}=2.3$ and the normalization coefficient as $b=\frac{\left.4 \overline{\mid P B}\right|^{2} \bar{D}}{\underline{\lambda}}=3200$. We show simulation results for $X_{1}(0)=X_{2}(0) \stackrel{\underline{\lambda}}{=} 0.5, \hat{\theta}(0)=0$, and two different values of $\hat{D}(0)$.
Figure 1 shows that, as Theorem 1 predicts, perfect asymptotic tracking is achieved (in a single simulation) for the output, state, and input, despite starting with an initial delay estimate of $\hat{D}(0)=0$, namely, for a large parametric uncertainty and with no predictor feedback initially. Figure 2 shows two distinct simulations, starting from two extreme initial values for the delay estimate, one at zero, and the other at twice the true delay value. The dashed simulation starts with a predictor feedback that initially overcompensates the delay, but it later adjusts the value of the delay estimate to obtain a stabilizing value of this parameter in the predictor feedback.

In Figure 2 we observe that $\hat{\theta}(t)$ converges to the true $\theta$ in both simulations, whereas this is not the case with $\hat{D}(t)$. This is consistent with the theory. By examining the error systems (44), (45), with the help of a persistency of excitation argument, we could infer the convergence of $\hat{\theta}(t)$ but not of $\hat{D}(t)$.

\section{Proof of the Main Result}

In this section, we prove Theorem 1 . We start by considering the transformation (38) along with its inverse

$$
\begin{aligned}
e(x, t)= & w(x, t)+\hat{D}(t) \int_{0}^{x} K(\hat{\theta}) \mathrm{e}^{(A+B K)(\hat{\theta}) \hat{D}(t)(x-y)} B(\hat{\theta}) \\
& \times w(y, t) d y+K(\hat{\theta}) \mathrm{e}^{(A+B K)(\hat{\theta}) \hat{D}(t) x} \tilde{X}(t) .
\end{aligned}
$$

Using these transformations and the models (1) and (18), the transformed system is written as

$$
\begin{aligned}
\dot{\tilde{X}}(t)= & (A+B K)(\hat{\theta}) \tilde{X}(t)+B(\hat{\theta}) w(0, t)+A(\tilde{\theta}) X(t) \\
& +B(\tilde{\theta}) u(0, t)-\frac{\partial X^{r}}{\partial \hat{\theta}}(t, \hat{\theta}) \dot{\hat{\theta}}(t) \\
D w_{t}(x, t)= & w_{x}(x, t)-\tilde{D}(t) p_{0}(x, t)-D \dot{\hat{D}}(t) q_{0}(x, t) \\
& -D \tilde{\theta}(t)^{T} p(x, t)-D \dot{\hat{\theta}}(t)^{T} q(x, t) \\
w(1, t)= & 0
\end{aligned}
$$

where $\tilde{D}(t)=D-\hat{D}(t)$ is the estimation error of the delay, the quantities

$$
\begin{aligned}
& A(\tilde{\boldsymbol{\theta}})=\sum_{i=1}^{p} \tilde{\theta}_{i} A_{i}=\sum_{i=1}^{p}\left(\theta_{i}-\hat{\theta}_{i}(t)\right) A_{i} \\
& B(\tilde{\boldsymbol{\theta}})=\sum_{i=1}^{p} \tilde{\theta}_{i} B_{i}
\end{aligned}
$$

are linear in the parameter estimation error $\tilde{\theta}(t)=\theta-\hat{\theta}(t)$, and

$$
\begin{aligned}
p_{0}(x, t)= & K(\hat{\theta}) \mathrm{e}^{A(\hat{\theta}) \hat{D}(t) x}((A+B K)(\hat{\theta}) \tilde{X}(t) \\
& +B(\hat{\theta}) w(0, t)) \\
q_{0}(x, t)= & \int_{0}^{x} K(\hat{\theta})(I+A(\hat{\theta}) \hat{D}(t)(x-y)) \mathrm{e}^{A(\hat{\theta}) \hat{D}(t)(x-y)}
\end{aligned}
$$




$$
\begin{aligned}
& \times B(\hat{\theta}) e(y, t) d y+K(\hat{\theta}) A(\hat{\theta}) x \mathrm{e}^{A(\hat{\theta}) \hat{D}(t) x} \tilde{X}(t) \\
= & \int_{0}^{x} w(y, t)[K(\hat{\theta})(I+A(\hat{\theta}) \hat{D}(t)(x-y)) \\
& \times \mathrm{e}^{A(\hat{\theta}) \hat{D}(t)(x-y)} B(\hat{\theta})+\hat{D}(t) \int_{y}^{x} K(\hat{\theta}) \\
& \times(I+A(\hat{\theta}) \hat{D}(t)(x-\xi)) \mathrm{e}^{A(\hat{\theta}) \hat{D}(t)(x-\xi)} B K(\hat{\theta}) \\
& \left.\times \mathrm{e}^{(A+B K)(\hat{\theta}) \hat{D}(t)(\xi-y)} B(\hat{\theta}) d \xi\right] d y+[K A(\hat{\theta}) x \\
& \times \mathrm{e}^{A(\hat{\theta}) \hat{D}(t) x}+\int_{0}^{x} K(\hat{\theta})(I+A(\hat{\theta}) \hat{D}(t)(x-y)) \\
& \left.\times \mathrm{e}^{A(\hat{\theta}) \hat{D}(t)(x-y)} B K(\hat{\theta}) \mathrm{e}^{(A+B K)(\hat{\theta}) \hat{D}(t) y} d y\right] \tilde{X}(t) .(5
\end{aligned}
$$

The vector-valued functions $q(x, t)$ and $p(x, t)$ are defined through their coefficients as follows, for $1 \leq i \leq p$,

$$
\begin{aligned}
p_{i}(x, t)= & K(\hat{\theta}) \mathrm{e}^{A(\hat{\theta}) \hat{D}(t) x}\left(A_{i} X(t)+B_{i} u(0, t)\right) \\
= & K(\hat{\theta}) \mathrm{e}^{A(\hat{\theta}) \hat{D}(t) x}\left(\left(A_{i}+B_{i} K(\hat{\theta})\right) \tilde{X}(t)+B_{i} w(0, t)\right. \\
& \left.+A_{i} X^{r}(t, \hat{\theta})+B_{i} u^{r}(0, t, \hat{\theta})\right) \\
q_{i}(x, t)= & \hat{D}(t) \int_{0}^{x}\left(\left[\frac{\partial K}{\partial \hat{\theta}_{i}}(\hat{\theta})+K(\hat{\theta}) A_{i} \hat{D}(t)(x-y)\right]\right. \\
& \left.\times \mathrm{e}^{A(\hat{\theta}) \hat{D}(t)(x-y)} B(\hat{\theta})+K(\hat{\theta}) \mathrm{e}^{A(\hat{\theta}) \hat{D}(t)(x-y)} B_{i}\right) \\
& \times e(y, t) d y+\left(\frac{\partial K}{\partial \hat{\theta}_{i}}+K(\hat{\theta}) A_{i} \hat{D}(t) x\right) \mathrm{e}^{A(\hat{\theta}) \hat{D}(t) x} \\
& \times \tilde{X}(t)-K(\hat{\theta}) \mathrm{e}^{A(\hat{\theta}) \hat{D}(t) x} \frac{\partial X^{r}}{\partial \hat{\theta}_{i}}(t, \hat{\theta})+\frac{\partial u^{r}}{\partial \hat{\theta}_{i}}(x, t, \hat{\theta}) \\
& -\hat{D}(t) \int_{0}^{x} K(\hat{\theta}) \mathrm{e}^{A(\hat{\theta}) \hat{D}(t)(x-y)} B(\hat{\theta}) \\
& \times \frac{\partial u^{r}}{\partial \hat{\theta}_{i}}(y, t, \hat{\theta}) d y \\
= & \hat{D}(t) \int_{0}^{x} w(y, t)\left[\left(\frac{\partial K}{\partial \hat{\theta}_{i}}(\hat{\theta})+K(\hat{\theta}) A_{i} \hat{D}(t)(x-y)\right)\right. \\
& \times \mathrm{e}^{A(\hat{\theta}) \hat{D}(t)(x-y)} B(\hat{\theta})+K(\hat{\theta}) \mathrm{e}^{A(\hat{\theta}) \hat{D}(t)(x-y)} B_{i} \\
& +\hat{D}(t) \int_{y}^{x}\left(\left(\frac{\partial K}{\partial \hat{\theta}_{i}}(\hat{\theta})+K(\hat{\theta}) A_{i} \hat{D}(t)(x-\xi)\right)\right. \\
& \left.\times \mathrm{e}^{A(\hat{\theta}) \hat{D}(t)(x-\xi)} B(\hat{\theta})+K(\hat{\theta}) \mathrm{e}^{A(\hat{\theta}) \hat{D}(t)(x-\xi)} B_{i}\right) \\
& \left.\times K(\hat{\theta}) \mathrm{e}^{(A+B K)(\hat{\theta}) \hat{D}(t)(\xi-y)} B(\hat{\theta}) d \xi\right] d y \\
& +\left[\left(\frac{\partial K}{\partial \hat{\theta}_{i}}(\hat{\theta})+K(\hat{\theta}) A_{i} \hat{D}(t) x\right) \mathrm{e}^{A(\hat{\theta}) \hat{D}(t) x}\right. \\
& +\hat{D}(t) \int_{0}^{x}\left[\left(\frac{\partial K}{\partial \hat{\theta}_{i}}(\hat{\theta})+K(\hat{\theta}) A_{i} \hat{D}(t)(x-y)\right)\right. \\
& \left.\times \mathrm{e}^{A(\hat{\theta}) \hat{D}(t)(x-y)} B(\hat{\theta})+K(\hat{\theta}) \mathrm{e}^{A(\hat{\theta}) \hat{D}(t)(x-y)} B_{i}\right] \\
& \left.\times K(\hat{\theta}) \mathrm{e}^{(A+B K)(\hat{\theta}) \hat{D}(t) y} d y\right] \tilde{X}(t) \\
& -K(\hat{\theta}) \mathrm{e}^{A(\hat{\theta}) \hat{D}(t) x} \frac{\partial X^{r}}{\partial \hat{\theta}_{i}}(t, \hat{\theta})+\frac{\partial u^{r}}{\partial \hat{\theta}_{i}}(x, t, \hat{\theta}) \\
\hline & \int_{0}^{x} K(\hat{\theta}) \mathrm{e}^{A(\hat{\theta}) \hat{D}(x-y)} B(\hat{\theta}) \frac{\partial u^{r}}{\partial \hat{\theta}_{i}}(y, t, \hat{\theta}) d y(55) \\
&
\end{aligned}
$$

Now, we define the following Lyapunov function candidate

$$
V(t)=D \log (N(t))+\frac{b}{\gamma_{1}} \tilde{D}(t)^{2}+\frac{b D}{\gamma_{2}} \tilde{\boldsymbol{\theta}}(t)^{T} \tilde{\boldsymbol{\theta}}(t),
$$

where

$$
N(t)=1+\tilde{X}(t)^{T} P(\hat{\theta}) \tilde{X}(t)+b \int_{0}^{1}(1+x) w(x, t)^{2} d x .
$$

Taking a time derivative of $V(t)$, we obtain

$$
\begin{aligned}
\dot{V}(t)= & -\frac{2 b}{\gamma_{1}} \tilde{D}(t)\left(\dot{\hat{D}}(t)-\gamma_{1} \tau_{D}(t)\right) \\
& -\frac{2 b D}{\gamma_{2}} \tilde{\theta}(t)^{T}\left(\dot{\hat{\theta}}(t)-\gamma_{2} \tau_{\theta}(t)\right) \\
& +\frac{D}{N(t)}\left(\sum _ { i = 1 } ^ { p } \dot { \hat { \theta } } _ { i } ( t ) \left(\tilde{X}(t)^{T} \frac{\partial P}{\partial \hat{\theta}_{i}}(\hat{\theta}) \tilde{X}(t)\right.\right. \\
& \left.-\tilde{X}(t)^{T} P(\hat{\theta}) \frac{\partial X^{r}}{\partial \hat{\theta}_{i}}(t, \hat{\theta})\right)-\tilde{X}(t)^{T} Q(\hat{\theta}) \tilde{X}(t) \\
& +2 \tilde{X}(t)^{T} P(\hat{\theta}) B(\hat{\theta}) w(0, t)-\frac{b}{D}\|w\|^{2}-\frac{b}{D} w(0, t)^{2} \\
& -2 b \dot{\hat{D}}(t) \int_{0}^{1}(1+x) w(x, t) q_{0}(x, t) d x \\
& \left.-2 b \dot{\hat{\theta}}(t)^{T} \int_{0}^{1}(1+x) w(x, t) q(x, t) d x\right),
\end{aligned}
$$

where we have used an integration by parts. Using the assumptions that $\hat{D}(0) \in] 0 ; \bar{D}]$ and $\hat{\theta}(0) \in \Pi$, the update laws (32)-(33), and the properties of the projection operator, we get

$$
\begin{aligned}
\dot{V}(t) \leq & \frac{D}{N(t)}\left(\sum _ { i = 1 } ^ { p } \dot { \hat { \theta } } _ { i } ( t ) \left(\tilde{X}(t)^{T} \frac{\partial P}{\partial \hat{\theta}_{i}}(\hat{\theta}) \tilde{X}(t)\right.\right. \\
& \left.-\tilde{X}(t)^{T} P(\hat{\theta}) \frac{\partial X^{r}}{\partial \hat{\theta}_{i}}(t, \hat{\theta})\right)-\lambda_{\min }(Q(\hat{\theta}))|\tilde{X}(t)|^{2} \\
& +2 \tilde{X}(t)^{T} P(\hat{\theta}) B(\hat{\theta}) w(0, t)-\frac{b}{D}\|w\|^{2}-\frac{b}{D} w(0, t)^{2} \\
& -2 b \dot{\hat{D}}(t) \int_{0}^{1}(1+x) w(x, t) q_{0}(x, t) d x \\
& \left.-2 b \dot{\hat{\theta}}(t)^{T} \int_{0}^{1}(1+x) w(x, t) q(x, t) d x\right),
\end{aligned}
$$

and, substituting the expressions of (32) and (33) and using (39) with the Young inequality, we obtain

$$
\begin{aligned}
\dot{V}(t) \leq & -\frac{D}{2 N(t)}\left(\lambda_{\min }(Q)|\tilde{X}|^{2}+\frac{b}{D}\|w\|^{2}+2 \frac{b}{D} w(0, t)^{2}\right) \\
& +2 D b \gamma_{1} \frac{\int_{0}^{1}(1+x)\left|w(x, t) \| p_{0}(x, t)\right| d x}{N(t)} \\
& \times \frac{\int_{0}^{1}(1+x)\left|w(x) \| q_{0}(x, t)\right| d x}{N(t)}
\end{aligned}
$$




$$
\begin{aligned}
& +D \gamma_{2} \sum_{i=1}^{p}\left(\frac{\int_{0}^{1}(1+x)|w(x, t)|\left|p_{i}(x, t)\right| d x}{N(t)}\right. \\
& \left.\frac{+2\left|\tilde{X}(t)^{T} P(\hat{\theta}) / b\right|\left|A_{i} X(t)+B_{i} u(0, t)\right|}{N(t)}\right) \\
& \times \frac{1}{N(t)}\left(\left|\tilde{X}(t)^{T} \frac{\partial P}{\partial \hat{\theta}_{i}}(\hat{\theta}) \tilde{X}(t)\right|+\mid \tilde{X}(t)^{T} P(\hat{\theta})\right. \\
& \left.\frac{\partial X^{r}}{\partial \hat{\theta}_{i}}(t, \hat{\theta})\left|+2 b \int_{0}^{1}(1+x)\right| w(x, t)|| q_{i}(x, t) \mid d x\right) .
\end{aligned}
$$

Fuhermore, each signal depending on $\hat{\theta}$, namely $A, B, K, P$, $\partial P / \partial \hat{\theta}_{i}, \partial X^{r} / \partial \hat{\theta}_{i}$ and $\partial u^{r} / \partial \hat{\theta}_{i}$, is given as continuous in $\hat{\theta}$. Since $\hat{\theta}$ remains in $\Pi$, a closed and bounded subset of $\mathbb{R}^{p}$, each signal is bounded in terms of $\hat{\theta}$ and admits a finite upper bound. We denote $M_{A}=\sup _{\hat{\theta} \in \Pi}|A(\hat{\theta})|$ and define $M_{P}, M_{B}, M_{K}, M_{A+B K}, M_{\partial K} / \partial \hat{\theta}$ similarly. Therefore, using Cauchy-Schwartz and Young inequalities, along with (49), (50), (52) and (54), we get

$$
\begin{aligned}
& \int_{0}^{1}(1+x)|w(x, t)|\left|p_{0}(x, t)\right| d x \\
& \leq M_{0}\left(|\tilde{X}(t)|^{2}+\|w(t)\|^{2}+w(0, t)^{2}\right) \\
& \int_{0}^{1}(1+x)|w(x, t)|\left|q_{0}(x, t)\right| d x \leq M_{0}\left(|\tilde{X}(t)|^{2}+\|w(t)\|^{2}\right) \\
& \left(\int_{0}^{1}(1+x)\left|w(x, t) \| p_{i}(x, t)\right| d x+2\left|\tilde{X}(t)^{T} P(\hat{\theta}) / b\right|\right. \\
& \left.\times\left|A_{i} X(t)+B_{i} u(0, t)\right|\right) \\
& \leq M_{i}\left(|\tilde{X}(t)|^{2}+\|w(t)\|^{2}+w(0, t)^{2}+\|w(t)\|\right) \\
& \int_{0}^{1}(1+x)\left|w(x, t) \| q_{i}(x, t)\right| d x \\
& \leq M_{i}\left(|\tilde{X}(t)|^{2}+\|w(t)\|^{2}+\|w(t)\|\right)
\end{aligned}
$$

where $M_{0}$ and $M_{i}(1 \leq i \leq p)$ are sufficiently large constants given by

$$
\begin{aligned}
M_{0}= & M_{K} \max \left\{M_{A+B K}+M_{A}, 2 M_{K}\left(\left(1+M_{A} \bar{D}\right)\right.\right. \\
& \left.\left.\times\left(M_{B}+M_{B} M_{K}(1+\bar{D})\right)+M_{A}\right)\right\} \mathrm{e}^{\left(M_{A}+M_{A+B K}\right) \bar{D}} \\
M_{i}= & \max \left\{\left|A_{i}\right|+\left|B_{i}\right| M_{K}+\left|B_{i}\right|+2 M_{P} / b,\right. \\
& 2 \sup _{(t, \hat{\theta}) \in \mathbb{R} \times \Pi}\left(\left|A_{i}\right|\left|X^{r}(t, \hat{\theta})\right|+\left|B_{i}\right|\left|u^{r}(0, t, \hat{\theta})\right|\right), \\
& 2 M_{K} \sup _{(t, \hat{\theta}) \in \mathbb{R} \times \Pi}\left|\frac{\partial X^{r}}{\partial \hat{\theta}}(t, \hat{\theta})\right|+2 \sup _{(t, \hat{\theta}) \in \mathbb{R} \times \Pi}\left|\frac{\partial u^{r}}{\partial \hat{\theta}}(t, \hat{\theta})\right| \\
& \times\left(1+\hat{D} M_{K} M_{B}\right),\left(\left(M_{\partial K / \partial \hat{\theta}}+M_{K}\left|A_{i}\right| \bar{D}\right) M_{B}+\left|A_{i}\right| M_{K}\right) \\
& \left.\times\left(2 \bar{D}+2 \bar{D} M_{K} M_{B}+M_{K}\right)\right\} \mathrm{e}^{\left(M_{A}+M_{A+B K}\right) \bar{D}} .
\end{aligned}
$$

Consequently, if we define

$$
\begin{aligned}
& M_{P}^{\prime}=\max _{1 \leq i \leq p} \sup _{\hat{\theta} \in \Pi}\left|\frac{\partial P(\hat{\theta})}{\partial \hat{\theta}_{i}}\right| \\
& M_{r}=\max _{1 \leq i \leq p} \sup _{\hat{\theta} \in \Pi, t \geq 0}\left|\frac{\partial X^{r}(t, \hat{\theta})}{\partial \hat{\theta}_{i}}\right|
\end{aligned}
$$

using (61)-(64) in (60), we obtain

$$
\begin{aligned}
\dot{V}(t) \leq & -\frac{D}{2 N(t)}\left(\lambda_{\min }(Q)|\tilde{X}|^{2}+\frac{b}{D}\|w\|^{2}+2 \frac{b}{D} w(0, t)^{2}\right) \\
& +2 D b \gamma_{1} M_{0}^{2} \frac{|\tilde{X}(t)|^{2}+\|w(t)\|^{2}+w(0, t)^{2}}{N(t)} \\
& \times \frac{|\tilde{X}(t)|^{2}+\|w(t)\|^{2}}{N(t)}+D \gamma_{2} \sum_{i=1}^{p} \frac{M_{i}}{N(t)}\left(|\tilde{X}(t)|^{2}+\|w(t)\|^{2}\right. \\
& \left.+w(0, t)^{2}+\|w(t)\|\right) \frac{1}{N(t)}\left(M_{P}^{\prime}|\tilde{X}(t)|^{2}+M_{r}|\bar{P} \| \tilde{X}(t)|\right. \\
& \left.+2 b M_{i}\left(|\tilde{X}(t)|^{2}+\|w(t)\|^{2}+\|w(t)\|\right)\right) .
\end{aligned}
$$

Bounding the cubic and quadric terms with the help of $N(t)$, we arrive at

$$
\begin{aligned}
\dot{V}(t) \leq & -\frac{D}{2 N(t)}\left(\underline{\lambda}|\tilde{X}(t)|^{2}+\frac{b}{D}\|w(t)\|^{2}+2 \frac{b}{D} w(0, t)^{2}\right) \\
& +\frac{2 D b \gamma_{1} M_{0}^{2}}{\min \{\underline{\lambda}, b\}} \frac{|\tilde{X}(t)|^{2}+\|w(t)\|^{2}+w(0, t)^{2}}{N(t)} \\
& +D \gamma_{2} \sum_{i=1}^{p} M_{i}\left(M_{P}^{\prime}\left(\frac{1}{\underline{\lambda}}+\frac{1}{2 \min \{1, b\}}\right)\right. \\
& +M_{P} M_{r}\left(\frac{1}{2}+\frac{1}{2 \min \{1, \underline{\lambda}\}}\right) \\
& \left.+2 b M_{i}\left(\frac{1}{\min \{\underline{\lambda}, b\}}+\frac{1}{2 \min \{1, b\}}+1\right)\right) \\
& \times \frac{|\tilde{X}(t)|^{2}+\|w(t)\|^{2}+w(0, t)^{2}}{N(t)} .
\end{aligned}
$$

Defining the following constant,

$$
m=\frac{2 \max \left\{b M_{0}^{2}, \sum_{i=1}^{p} M_{i}\left(M_{P}^{\prime}+M_{P} M_{r}+3 b M_{i}\right)\right\}}{\min \{1, \underline{\lambda}, b\}}
$$

we finally obtain

$$
\begin{aligned}
\dot{V}(t) \leq & -\frac{D}{2 N(t)}\left(\min \left\{\underline{\lambda}, \frac{b}{D}\right\}-2\left(\gamma_{1}+\gamma_{2}\right) m\right) \\
& \times\left(|\tilde{X}(t)|^{2}+\|w(t)\|^{2}+w(0, t)^{2}\right) .
\end{aligned}
$$

Consequently, by choosing

$$
\gamma^{*}=\frac{\min \{\underline{\lambda}, b / D\}}{4 b m}
$$


and $\left(\gamma_{1}, \gamma_{2}\right) \in\left[0 ; \gamma^{*}\left[{ }^{2}\right.\right.$, we make $\dot{V}(t)$ negative semidefinite and hence

$$
V(t) \leq V(0), \quad \forall t \geq 0 .
$$

Starting from this result, we now prove the results stated in Theorem 1. From the transformation (38) and its inverse (43), we obtain these two inequalities

$$
\begin{aligned}
\|w(t)\|^{2} & \leq r_{1}\|e(t)\|^{2}+r_{2}|\tilde{X}(t)|^{2} \\
\|e(t)\|^{2} & \leq s_{1}\|w(t)\|^{2}+s_{2}|\tilde{X}(t)|^{2},
\end{aligned}
$$

where $r_{1}, r_{2}, s_{1}, s_{2}$ are sufficiently large positive constants given by

$$
\begin{aligned}
& r_{1}=3\left(1+\bar{D}^{2} M_{K}^{2} \mathrm{e}^{2 M_{A+B K} \bar{D}} M_{B}^{2}\right) \\
& r_{2}=3 M_{K}^{2} \mathrm{e}^{2 M_{A+B K} \bar{D}} \\
& s_{1}=3\left(1+\bar{D}^{2} M_{K}^{2} \mathrm{e}^{2 M_{A} \bar{D}} M_{B}^{2}\right) \\
& s_{2}=3 M_{K}^{2} \mathrm{e}^{2 M_{A} \bar{D}} .
\end{aligned}
$$

Furthermore, from (56) and (76), it follows that

$$
\begin{aligned}
\tilde{D}(t)^{2}+\tilde{\theta}(t)^{T} \tilde{\theta}(t) & \leq \frac{\gamma_{1}+\gamma_{2}}{b} V(t) \\
\|\tilde{X}(t)\|^{2} & \leq \frac{1}{\lambda}\left(\mathrm{e}^{V(t) / D}-1\right) \\
\|e(t)\| & \leq \frac{\frac{s_{1}}{b}}{b}\left(\mathrm{e}^{V(t) / D}-1\right)+s_{2}\|\tilde{X}(t)\| .
\end{aligned}
$$

Thus, from the definition of $\Upsilon(t)$, it is easy to show that

$$
\Upsilon(t) \leq\left(\frac{1+s_{2}}{\underline{\lambda}}+\frac{s_{1}}{b}+\frac{\left(\gamma_{1}+\gamma_{2}\right) D}{b}\right)\left(\mathrm{e}^{V(t) / D}-1\right)
$$

Besides, using (75), we also obtain

$$
V(0) \leq\left(D\left(\bar{\lambda}+s_{2} b+2 s_{1} b\right)+b\left(\frac{1}{\gamma_{1}}+\frac{1}{\gamma_{2}}\right)\right) \Upsilon(0) .
$$

Finally, if we define

$$
\begin{aligned}
& R=\frac{1+s_{2}}{\underline{\lambda}}+\frac{s_{1}}{b}+\frac{\left(\gamma_{1}+\gamma_{2}\right) D}{b} \\
& \rho=\bar{\lambda}+s_{2} b+2 s_{1} b+\frac{b}{D}\left(\frac{1}{\gamma_{1}}+\frac{1}{\gamma_{2}}\right),
\end{aligned}
$$

we obtain the global stability result given in Theorem 1 .

We now proove tracking. From (74), we obtain the uniform boudedness of $\|\tilde{X}(t)\|,\|w(t)\|, \hat{D}(t)$ and $\|\hat{\boldsymbol{\theta}}(t)\|$. From (43), we obtain that $\|e(t)\|$ is also uniformly bounded in time. From (31), we get the uniformly boudedness of $U(t)$ and consequently of $\tilde{U}(t)$ for $t \geq 0$. Thus, we get that $u(0, t)$ and $e(0, t)$ are uniformly bounded for $t \geq D$. Besides, from
(33) and (52), we obtain the uniform boundedness of $\|\dot{\hat{\theta}}(t)\|$ for $t \geq D$. Finally, with (44), we obtain that $d \tilde{X}(t)^{2} / d t$ is uniformly bounded for $t \geq D$. As $|\tilde{X}(t)|$ is square integrable, from (72), we conclude from Barbalat's Lemma that $\tilde{X}(t) \rightarrow$ 0 when $t \rightarrow \infty$.

Besides, from (72), we get the square integrability of $\|w(t)\|$. From (76), we obtain the square integrability of $\|e(t)\|$. Consequently, with (31), we obtain the square integrability of $\tilde{U}(t)$. Furthermore,

$$
\begin{aligned}
\frac{d \tilde{U}(t)^{2}}{d t}= & 2 \tilde{U}(t)\left(K(\hat{\theta}) \mathrm{e}^{A(\hat{\theta}) \hat{D}(t)} \dot{\tilde{X}}(t)+\dot{\hat{D}}(t) G_{0}(t)\right. \\
& \left.+\sum_{i=1}^{p} \dot{\hat{\theta}}_{i}(t) G_{i}(t)+\frac{\hat{D}}{D} H(t)\right)
\end{aligned}
$$

with

$$
\begin{aligned}
G_{0}(t)= & K(\hat{\theta})\left[A(\hat{\theta}) \mathrm{e}^{A(\hat{\theta}) \hat{D}(t)} \tilde{X}(t)+\int_{0}^{1}(I+A(\hat{\theta}) \hat{D}(t)\right. \\
& \left.\times(1-y)) \mathrm{e}^{A(\hat{\theta}) \hat{D}(t)(1-y)} B(\hat{\theta}) e(y, t) d y\right] \\
G_{i}(t)= & \frac{\partial K}{\partial \hat{\theta}}(\hat{\theta})\left[\mathrm{e}^{A(\hat{\theta}) \hat{D}(t)} \tilde{X}(t)+\hat{D}(t) \int_{0}^{1} \mathrm{e}^{A(\hat{\theta}) \hat{D}(t)(1-y)}\right. \\
& \times B(\hat{\theta}) e(y, t) d y]+K(\hat{\theta})\left[A_{i} \hat{D}(t) \mathrm{e}^{A(\hat{\theta}) \hat{D}(t)} \tilde{X}(t)\right. \\
& +\hat{D}(t) \int_{0}^{1}\left[A_{i} \hat{D}(t)(1-y) \mathrm{e}^{A \hat{D}(t)(1-y)} B(\hat{\theta})\right. \\
& \left.\left.+\mathrm{e}^{A \hat{D}(t)(1-y)} B_{i}\right] e(y, t) d y\right] \\
H(t)= & K(\hat{\theta})\left[B(\hat{\theta}) \tilde{U}(t)-\mathrm{e}^{A(\hat{\theta}) \hat{D}(t)} B(\hat{\theta}) e(0, t)\right. \\
& \left.+\int_{0}^{1} A(\hat{\theta}) \hat{D}(t) \mathrm{e}^{A(\hat{\theta}) \hat{D}(t)(1-y)} B(\hat{\theta}) e(y, t) d y\right] .
\end{aligned}
$$

The signals $\dot{\hat{D}}, \dot{\hat{\theta}}_{1}, \ldots, \dot{\hat{\theta}}_{p}$ are uniformly bounded over $t \geq 0$, according to (32)-(33). By using the uniform boundedness of $\tilde{X}(t), \dot{\tilde{X}}(t),\|e(t)\|, \tilde{U}(t)$ over $t \geq 0$ and of $e(0, t)$ for $t \geq D$ and the uniform boundedness of all the signals which are functions of $\hat{\theta}$ for $t \geq 0$, we obtain the uniform boundedness of $d \tilde{U}(t)^{2} / d t$ over $t \geq D$. Then, with Barbalat's lemma, we conclude that $\tilde{U}(t) \rightarrow 0$ when $t \rightarrow \infty$.

\section{Conclusions}

In this paper we presented a result on global adaptive tracking for ODEs with unknown parameters and long unknown actuator delays, with full state feedback. The control design employs predictor feedback, modified from stabilization to tracking, whereas adaptation employs our relatively novel Lyapunov update law design with normalization. A simulation example with a second order linear plant illustrates the effectiveness of the design.

Future work should focus on systems with simultaneous state an input delay. The non-adaptive design (Jankovic 2008) 
may be a good starting point for an interesting class of systems, and the clf tools (Karafyllis and Jiang 2008) for systems with state delays may be instrumental.

\section{References}

Z. Artstein, "Linear systems with delayed controls: a reduction," IEEE Trans. on Automatic Control, 27, pp. 869-879, 1982.

D. Bresch-Pietri and M. Krstic, "Delay-adaptive full-state predictor feedback for systems with unknown long actuator delay," 2009 American Control Conference.

S. Diop, I. Kolmanovsky, P. E. Moraal, and M. van Nieuwstadt, "Preserving stability/performance when facing an unknown timedelay," Control Engineering Practice, vol. 9, pp. 1319-1325, 2001.

S. Evesque, A. M. Annaswamy, S. Niculescu, and A. P. Dowling, "Adaptive Control of A Class of Time-delay Systems," ASME Transactions on Dynamics, Systems, Measurement, and Control, vol. 125, pp. 186-193, 2003.

Y. A. Fiagbedzi and A. E. Pearson, "Feedback stabilization of linear autonomous time lag systems," IEEE Trans. Automatic Control, 31, pp. 847-855, 1986.

K. Gu and S.-I. Niculescu, "Survey on recent results in the stability and control of time-delay systems," Transactions of ASME, 125, pp. 158165,2003

M. Jankovic, "Control of cascade systems with time delay-the integral cross-term approach," 2006 IEEE Conference on Decision and Control.

M. Jankovic, "Forwarding, backstepping, and finite spectrum assignment for time delay systems," 2006 American Control Conference.

M. Jankovic, "Recursive predictor design for linear systems with time delay," 2008 American Control Conference.

I. Karafyllis and Z. P. Jiang, "Control Lyapunov functionals and robust stabilization of nonlinear time-delay systems," Proc. IEEE. Conf. Decision Contr., pp. 5312-5317, 2008.

J. Klamka, "Observer for linear feedback control of systems with distributed delays in controls and outputs," Syst Contr Lett, 1, pp. 326331, 1982.

M. Krstic, "Lyapunov tools for predictor feedbacks for delay systems: Inverse optimality and robustness to delay mismatch," Automatica, vol. 44, pp. 2930-2935, 2008

M. Krstic, "On compensating long actuator delays in nonlinear control," IEEE Transactions on Automatic Control, vol. 53, pp. 1684-1688, 2008.

M. Krstic and A. Banaszuk, "Multivariable adaptive control of instabilities arising in jet engines," Control Engineering Practice, vol. 14, pp. 833-842, 2006.

M. Krstic, I. Kanellakopoulos, and P. Kokotovic, Nonlinear and Adaptive Control Design, Wiley, New York, 1995.

M. Krstic and A. Smyshlyaev, "Adaptive boundary control for unstable parabolic PDEs-Part I: Lyapunov design," IEEE Transactions on Automatic Control, vol. 53, pp. 1575-1591, 2008.

M. Krstic and A. Smyshlyaev, "Backstepping boundary control for first order hyperbolic PDEs and application to systems with actuator and sensor delays," Systems \& Control Letters, vol. 57, pp. 750-758, 2008.

M. Krstic and A. Smyshlyaev, Boundary Control of PDEs: A Course on Backstepping Designs, SIAM, 2008.

W. H. Kwon and A. E. Pearson, "Feedback stabilization of linear systems with delayed control," IEEE Trans. on Automatic Control, 25, pp. 266-269, 1980.
A. Z. Manitius and A. W. Olbrot, "Finite spectrum assignment for systems with delays," IEEE Trans. on Automatic Control, 24, pp. 541-553, 1979.

W. Michiels and S.-I. Niculescu, "On the delay sensitivity of Smith predictors," International Journal of Systems Science, vol. 34, pp. 543-551, 2003.

W. Michiels and S.-I. Niculescu, Stability and Stabilization of Time-Delay Systems: An Eignevalue-Based Approach, SIAM, 2007.

L. Mirkin, "On the approximation of distributed-delay control laws," Systems \& Control Letters, vol. 51, pp. 331-342, 2004.

S. Mondie and W. Michiels, "Finite spectrum assignment of unstable time-delay systems with a safe implementation," IEEE Trans. on Automatic Control, 48, pp. 2207-2212, 2003.

S.-I. Niculescu and A. M. Annaswamy, "An Adaptive Smith-Controller for Time-delay Systems with Relative Degree $n^{*} \geq 2$," Systems \& Control Letters, vol. 49, pp. 347-358, 2003.

A. W. Olbrot, "Stabilizability, detectability, and spectrum assignment for linear autonomous systems with general time delays," IEEE Trans. on Automatic Control, 23, pp. 887-890, 1978.

R. Ortega and R. Lozano, "Globally stable adaptive controller for systems with delay," Internat. J. Control, vol. 47, pp. 17-23, 1988.

J.-P. Richard, "Time-delay systems: An overview of some recent advances and open problems," Automatica, vol. 39, pp. 1667-1694, 2003.

A. Smyshlyaev and M. Krstic, "Closed form boundary state feedbacks for a class of 1D partial integro-differential equations," IEEE Trans. on Automatic Control, Vol. 49, No. 12, pp. 2185-2202, 2004.

A. Smyshlyaev and M. Krstic, "Backstepping observers for a class of parabolic PDEs," Systems and Control Letters, vol.54, pp. 613-625, 2005.

G. Tadmor, "The standard $H_{\infty}$ problem in systems with a single input delay," IEEE Transactions on Automatic Control, vol. 45, pp. 382397,2000

K. Watanabe and M. Ito, "An observer for linear feedback control laws of multivariable systems with multiple delays in controls and outputs," Systems and Control Letters, 1, pp. 54-59, 1981.

K. Watanabe, "Finite spectrum assignment and observer for multivariable systems with commensurate delays," IEEE Transactions on Automatic Control, vol. 31, pp. 543-550, 1996.

L. Zaccarian and D. Nesic, "A cascade interpretation of the Smith predictor and the arising enhanced scheme," 2006 American Control Conference.

Q.-C. Zhong, "On distributed delay in linear control laws-Part I: Discrete-delay implementation," IEEE Transactions on Automatic Control, vol. 49, pp. 2074-2080, 2006.

Q.-C. Zhong, Robust Control of Time-delay Systems, Springer, 2006.

Q.-C. Zhong and L. Mirkin, "Control of integral processes with dead time-Part 2: Quantitative analysis," IEE Proc. Control Theory \& Appl. , vol. 149, pp. 291-296, 2002

J. Zhou, W. Wang, and C. Wen, "Adaptive backstepping control of uncertain systems with unknown input time delay," Proc. 17th IFAC World Congress, pp. 13361-13366, Seoul, Korea, 2008. 


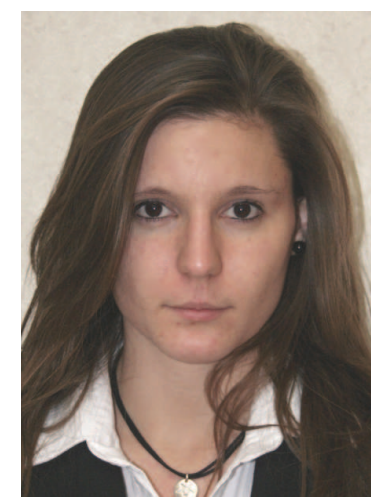

Delphine Bresch-Pietri is a graduate student Ecole des Mines de Paris, also known as Mines ParisTech, France, graduating with a Master degree in automatic control in 2009. She was a visiting student at University of California, San Diego, in summer of 2008 .

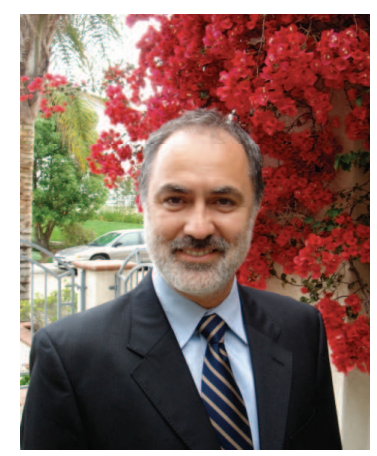

Miroslav Krstic is the Sorenson Distinguished Professor and the founding Director of the Cymer Center for Control Systems and Dynamics (CCSD) at UC San Diego. He received his PhD in 1994 from UC Santa Barbara and was Assistant Professor at University of Maryland until 1997. He is a coauthor of eight books: Nonlinear and Adaptive Control Design (Wiley, 1995), Stabilization of Nonlinear Uncertain Systems (Springer, 1998), Flow Control by Feedback (Springer, 2002), Real-time Optimization by Extremum Seeking Control (Wiley, 2003), Control of Turbulent and Magnetohydrodynamic Channel Flows (Birkhauser, 2007), Boundary Control of PDEs: A Course on Backstepping Designs (SIAM, 2008), Delay Compensation for Nonlinear, Adaptive, and PDE Systems (Birkhauser, 2009), and Adaptive Control of Parabolic PDEs (Princeton, 2009). Krstic is a Fellow of IEEE and IFAC and has received the Axelby and Schuck paper prizes, NSF Career, ONR Young Investigator, and PECASE award. He has held the appointment of Springer Distinguished Visiting Professor of Mechanical Engineering at UC Berkeley. 